\title{
Presse et Plumes. Journalisme et littérature au XIX siècle, sous la direction de Marie-Ève Therenty et Alain Vaillant
}

\section{Michel Arrous}

\section{OpenEdition}

Journals

\section{Édition électronique}

URL : http://journals.openedition.org/studifrancesi/5651

DOI : ERREUR PDO dans /localdata/www-bin/Core/Core/Db/Db.class.php L.34 : SQLSTATE[HY000]

[2006] MySQL server has gone away

ISSN : 2427-5856

\section{Éditeur}

Rosenberg \& Sellier

Édition imprimée

Date de publication : 1 septembre 2011

Pagination : 416-418

ISSN : 0039-2944

Référence électronique

Michel Arrous, «Presse et Plumes. Journalisme et littérature au xix ${ }^{\mathrm{e}}$ siècle, sous la direction de Marie-Ève Therenty et Alain Vaillant », Studi Francesi [En ligne], 164 (LV | II) | 2011, mis en ligne le 30 novembre 2015, consulté le 07 janvier 2021. URL : http://journals.openedition.org/studifrancesi/5651 ; DOI : https://doi.org/10.4000/studifrancesi.5651

Ce document a été généré automatiquement le 7 janvier 2021.

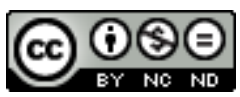

Studi Francesi è distribuita con Licenza Creative Commons Attribuzione - Non commerciale - Non opere derivate 4.0 Internazionale. 


\title{
Presse et Plumes. Journalisme et littérature au XIX $\mathrm{X}^{\mathrm{e}}$ siècle, sous la direction de Marie-Ève Therenty et Alain Vaillant
}

\author{
Michel Arrous
}

\section{RÉFÉRENCE}

AA. VV., Presse et Plumes. Journalisme et littérature au XIX $X^{\mathrm{e}}$ siècle, sous la direction de Marie-Ève THERENTY et Alain VAILlANT, Paris, Nouveau Monde éditions, 2004, 583 pp.

1 Après 1836. L'An I de l'ère médiatique, ce volume est le bilan provisoire d'une recherche sur les rapports souvent conflictuels entre presse et littérature, de la Restauration à la fin du siècle. Quatre ensembles: «La presse au miroir de la littérature» détaille les complexes représentations que la littérature donne du journal; «Le journal entre provinces et nations» est consacré au journal à vocation locale qui se fait chambre d'écho de la révolution romantique et bientôt organe du mouvement régionaliste; «La création intellectuelle et littéraire au rythme du périodique» témoigne de l'influence du débat intellectuel sur la littérature et l'invention poétique; «Poétiques de l'écriture journalistique» pointe la mutation générique provoquée par les contraintes propres au nouveau média. Dans cette section, on se limitera aux études sur le premier dixneuvième siècle, début de l'ère médiatique, avant que ne commence l' «âge de papier», évoqué par le fameux dessin de F. Valloton publié peu après J'accuse...!

2 Avec Stendhal face à la presse de son temps (pp. 17-29), Béatrice DIAZ rappelle que ce grand dévoreur de journaux qui joua au «journaliste anglais» a donné une véritable radioscopie de la presse et du champ littéraire français sous la Restauration. En dénonçant la camaraderie et le charlatanisme sévissant dans le petit monde des journalistes «maîtres du jeu littéraire», Stendhal plaide pour un autre journalisme 
fondé sur des exigences d'invention et de courage. Dans L'esprit sous presse. Le journal et le journaliste selon la «littérature panoramique» (1781-1843) (pp. 31-50, avec bibliographie), José-Luis DIAZ suit les évolutions de la presse, à la fois sacerdoce et industrie, dans ce que W. Benjamin a appelé la «littérature panoramique» dont le modèle initial est le Tableau de Paris de Mercier. Première réhabilitation avec Étienne de Jouy, puis apologie du journaliste par Planche et Janin dans Paris ou le Livre des Cent et un (1832) et dans Les Français peints par eux-mêmes (1840), alors que Balzac vient d'en faire un portrait au vitriol dont les traits principaux se retrouveront dans les Mours des diurnales de Marcel Schwob en 1903. Janin s'emploiera en 1843 à contrer la satire "dévastatrice» de la Monographie de la presse parisienne dont les arguments étaient déjà en partie ceux de Mercier ou de la préface de Mademoiselle de Maupin. Mais si Balzac passe en revue les tares du journal, en même temps qu'il dénonce le gouffre de la presse, il est séduit par l'écriture journalistique.

3 L'aspect régional ou provincial est illustré par les cas des poètes Victor Pavie, à Angers, Charles Brugnot à Dijon, et celui d'Émile Souvestre à Nantes. Vincent LAISNEY (Victor Pavie et le "Feuilleton des Affiches d'Angers", pp. 95-104), retrace le parcours de ce poète sans originalité qui fut un familier du Cénacle et le principal rédacteur d'une modeste feuille de province dans laquelle il défendit la cause romantique. Son premier article, qui ne plut guère au lectorat local, retint l'attention de V. Hugo dont le groupe ne disposait plus de «La Muse française»; attention qu'on pourrait attribuer uniquement à l'«engouement soudain des romantiques pour la province», mais qui, à y regarder de près, révèle «l'attitude ambiguë du Cénacle vis à vis de la province»: les romantiques de la rue Notre-Dame-des-Champs, même s'ils sont séduits par la province, restent attachés à la capitale. Pavie rencontrera le Maître en 1827 - «le jeune Angevin ne s'en remettra jamais»-, séjournera quelque temps à Paris en 1829 pour, finalement, retrouver la "pauvre France provinciale», lui dira Hugo. La renaissance régionale des années 1820-1830 est aussi illustrée par une éphémère publication dijonnaise dont un des mérites fut d'accueillir les premières «bambochades» du futur Aloysius Bertrand. Jacques-Rémi DAHAN, dans Les correspondants parisiens du "Provincial de Dijon": collaboration ou instrumentalisation? (pp. 105-119), expose comment ce périodique, qui se référait à l'Académie provinciale, fondée à Lyon en 1826 sous l'égide de Chateaubriand, établit des liens réels ou fictifs avec trois notabilités parisiennes: son fondateur n'hésita pas à publier une lettre de Nodier, en fait un fort bon pastiche qui ne contribua sans doute pas à convaincre l'écrivain de collaborer. L'anti-centralisme de façade de Hugo, lequel «usa du "Provincial" comme d'une tribune commode» mais n'y signa pas la moindre ligne, confirme que l'intelligentsia parisienne «ne se soucie de la province que pour la vampiriser». En revanche, Chateaubriand «semble avoir fait preuve d'un relatif désintéressement» en n'instrumentalisant pas le «Provincial» où il trouvait un écho de sa pensée politique. Barbel PLÖTNER a choisi un écrivain moins obscur quoique longtemps négligé: en dépit de sa brièveté, Du «Lycée armoricain» à la «Mosaïque de l'Ouest»: Émile Souvestre, journaliste breton (pp.121-133) cite plusieurs publications importantes à travers leurs fondateurs et rédacteurs, tous attachés à leur province et liés à des groupes ou des organes parisiens («Le Globe» de Dubois, puis celui de Leroux, le «Magasin pittoresque» d'Édouard Charton). Souvestre illustre à lui seul l'influence des libéraux, des saint-simoniens et des républicains sur le journalisme breton. Autre apport important, celui de Diana COOPER-RICHET (Presse en anglais et littérature, à Paris, dans la première moitié du XIXe siècle, pp. 153-168), consacré aux publications parisiennes de la dynastie Galignani qui offraient aux habitués de son cabinet de lecture un panorama 
fidèle de la production littéraire, scientifique et artistique de la Grande-Bretagne, mais aussi de l'étranger, sous la forme d'articles puisés dans les grandes publications telles «The Edinburgh Review», «The British Critic» et, plus tard, «The Athenaeum». Deux recueils nés sous la monarchie de Juillet fondent leur spécificité sur les illustrations: «Le Magasin pittoresque» lancé par Charton, et «L'Illustration» dont il eut aussi l'idée. Familière de son œuvre, Marie-Laure AURENCHE - Du «Magasin pittoresque» (1833) à "L'Illustration»: la naissance du nouvellisme illustré (pp. 169-184) - décrit ses pratiques journalistiques ainsi que son modèle rédactionnel repris à une autre échelle par «L'Illustration», hebdomadaire d'actualités et d'informations, alors que le «Magasin» se voulait un recueil encyclopédique, ce qui supprima toute concurrence. Avec 1848 et "L'Illustration». La double naissance du reportage illustré et de la poste-réception des gravures (pp. 185-194) de Jean-Pierre ВАсот, on a un bel exemple de ce type d'information car les événement violents de 48 eurent une influence déterminante sur le journalisme qui mettra désormais en scène la «chose vue» par une gravure légendée. Cette tendance se développera au cours des années 1880, avec l'apparition des agences, jusqu'à devenir «l'universel reportage» qui tombera sous le mépris de Mallarmé.

Dans la troisième partie centrée sur le régime culturel du périodique - comme on dit le politique -, on retiendra les éclaircissements qu'apporte Philippe RÉGNIER sur la Pratique et [la] théorie saint-simonienne de la presse (pp. 224-239), autour de 1830 et jusqu'en 1870: une nouvelle philosophie de la presse caractérisée par la stratégie collective du «Globe», jusqu'en avril 1832, sous la conduite du Père Chevalier, à la tête des «hommes positifs» qui constituent "une très efficace école de journalisme» dont plusieurs éléments essaimeront vers «Le Temps», les «Débats», la «Revue encyclopédique». Cette diffusion posthume du saint-simonisme par les revues est un phénomène qu'on retrouve avec le positivisme après la mort de Comte qui, fort méfiant à l'égard de la presse, avait opté pour les cours publics, nous rappelle Annie PETIT (La presse positiviste au XIX ${ }^{e}$ siècle, pp. 273-291). Dans Journalisme et polémique religieuse: «L'Univers» et «L'Événement» (pp. 241-259), Jean-Claude FIZAINE analyse le conflit haineux déclenché par Veuillot contre «L'Événement», journal fondé par Meurice, Vacquerie et les fils de V. Hugo, au moment où le poète fit sa proposition de loi sur la misère et, surtout, pendant le vote de la loi Falloux (1849-1851); analyse poursuivie par Michèle FIZAINE (Procès de presse en 1850-1851: la défense de la littérature par Victor Hugo, pp. 261-272). «L'Événement», victime dès avant le coup d'État d'une série de poursuites, représente le romantisme politique et défend la liberté d'écrire au moment où la littérature, mise en cause parce qu'elle colle à la réalité, est accusée et condamnée pour «crime de feuilleton».

5 Le $\mathrm{XIX}^{\mathrm{e}}$ siècle a vu naître la plupart des grands genres journalistiques et a connu des innovations essentielles dans l'écriture du journal. À partir d'un corpus de périodiques libéraux qui auraient pu être plus exploités, Corinne PELTA ( $L a$ presse libérale sous la Restauration: Émergence d'une écriture collective, pp. 371-378) se livre à une analyse trop rapide de ce type d'écriture dont le caractère répétitif a pour but de souligner la force de l'opinion publique. Autre exemple de pratique, cette fois bien connu des historiens, le «Moniteur». En dépit de sa vocation à reproduire les actes du gouvernement, ce véritable mythe journalistique eut aussi à voir avec la littérature, particulièrement en son âge d'or, le Second Empire, avec Sainte-Beuve, Gautier, Mérimée ou Baudelaire. C'est ce que rappelle opportunément Laurence GUELLEC dans Un journal "monstre". Repères pour une histoire $d u$ «Moniteur universel» (1789-1868) (pp. 379-391), où est proposée 
une redéfinition du champ littéraire et culturel de cet outil de propagande qui fut aussi le «livre des orateurs» puisqu'il recueillait la transcription soigneusement arrangée des interventions à la Chambre. Cet aspect est souligné dans Presse, rhétorique, éloquence: Confrontations et reconfigurations (1830-1870) (pp. 393-414), par Corinne SAMINADAYARPERRIN qui voit justement dans la presse de ces années à la fois une espèce de conservatoire et un lieu de renouvellement du Discours public par le biais d'une éloquence en demi-teinte, et dans le journaliste un orateur, constat déjà fait par Balzac: «Bossuet serait aujourd'hui journaliste». Dans L'invention de la fiction d'actualité (pp. 415-427), Marie-Ève THÉRENTY traite d'une autre nouveauté dans la presse autour de 1830: la fiction brève insérée dans l'actualité, fiction ou conte d'actualité produit par des littérateurs qui sont autant d'«actualistes» et qui s'appellent Paul de Kock, Scribe, Janin, Berthoud, et même Balzac ou Sand. Au chapitre des nouveautés, il faut ajouter «Le Journal des enfants» de Lautour-Mézeray dont une partie de la longue carrière est retracée par Francis MARCOIN (La presse enfantine, de la Restauration à la monarchie de Juillet, pp. 429-441); et des pratiques d'écrivains-journalistes: celle de l' «homme de presse, homme de peine» que fut Gautier, écrivain-journaliste saisi dans son travail quotidien de 1837 à 1872, avec force précisions, par Patrick BERTHIER (Théophile Gautier: de quelques pratiques d'écriture, pp. 443-455); ou celle de Balzac: plus fort que Girardin, le romancier-réclamiste, aux prises avec la publicité, n'hésite pas à la faire entrer en littérature, ainsi que le rappelle plaisamment Véronique BUI (Comment l'huile céphalique vint à Balzac. Poétique de la publicité dans le quotidien, pp.457-466); ou encore celle de George Sand qui s'auto-instrumentalise en publiant dans la revue qu'elle fonde avec P. Leroux et L.Viardot. À propos de "Consuelo" dans "La Revue indépendante» (pp. 467-479), Christine PLANTÉ ne manque pas de rappeler que la romancière, même si cette alliance fut féconde, revendiqua bientôt l'autonomie du journal et de l'écrivain et souligna l'inconvénient de la publication en feuilleton à laquelle elle avait tenté de s'adapter. C'est à la critique journalistique, littéraire ou esthétique, que s'attache Marie-Françoise MELMOUX-MONTAUBIN ( «Contes de lettres» et écriture de soi: la critique littéraire dans le journal au XIXe siècle, pp. 481-498). Il s'agit d'une écriture marquée par la tactique du «détour», sous le signe de la fantaisie autobiographique qui peut prendre l'apparence de ce bavardage aimable par lequel le journaliste, Sainte-Beuve, Vallès ou France, devient écrivain. Procédé que Barbey récusait.

6 Avec cet ouvrage complété par une bibliographie et des index, on dispose d'un riche ensemble d'études sur les relations qu'entretiennent au XIX ${ }^{e}$ siècle l'imprimé périodique et la littérature. Le panorama n'est certes pas exhaustif - le rôle et l'apport de certaines publications pourtant souvent citées, entre autres «Le Globe» libéral, même si son magistère a été réévalué par les travaux de Jean-Jacques Goblot, la «Revue britannique» ou «L'Artiste»-, mais on a là une contribution décisive à l'histoire culturelle des poétiques. 